Article

\title{
Serum Catestatin Levels and Arterial Stiffness Parameters Are Increased in Patients with Inflammatory Bowel Disease
}

\author{
Piero Marin Zivkovic ${ }^{1,2}$, Andrija Matetic ${ }^{2,3}{ }^{(0)}$, Ivana Tadin Hadjina ${ }^{1}$, Doris Rusic ${ }^{4}$, \\ Marino Vilovic ${ }^{2}$, Daniela Supe-Domic ${ }^{5,6}$, Josip Andelo Borovac ${ }^{2}{ }^{(1)}$, Ivana Mudnic ${ }^{7}$, \\ Ante Tonkic ${ }^{1,8}$ and Josko Bozic ${ }^{2, *(D)}$ \\ 1 Department of Gastroenterology, University Hospital of Split, 21000 Split, Croatia; \\ piero.zivkovic@gmail.com (P.M.Z.); ihtadina@gmail.com (I.T.H.); jobo5k@yahoo.com (A.T.) \\ 2 Department of Pathophysiology, University of Split School of Medicine, 21000 Split, Croatia; \\ andrija.matetic@gmail.com (A.M.); marino.vilovic@mefst.hr (M.V.); josip.borovac@me.com (J.A.B.) \\ 3 Department of Cardiology, University Hospital of Split, 21000 Split, Croatia \\ 4 Department of Pharmacy, University of Split School of Medicine, 21000 Split, Croatia; doris.rusic@mefst.hr \\ 5 Department of Medical Laboratory Diagnostics, University Hospital of Split, 21000 Split, Croatia; \\ daniela.supedomic@gmail.com \\ 6 Department of Health Studies, University of Split, 21000 Split, Croatia \\ 7 Department of Pharmacology, University of Split School of Medicine, 21000 Split, Croatia; \\ ivana.mudnic@mefst.hr \\ 8 Department of Internal Medicine, University of Split School of Medicine, 21000 Split, Croatia \\ * Correspondence: josko.bozic@mefst.hr; Tel.: +385-21-557-871; Fax: +385-21-557-905
}

Received: 15 January 2020; Accepted: 25 February 2020; Published: 26 February 2020

\begin{abstract}
Catestatin (CST) is an important peptide in the pathophysiology of chronic inflammatory disorders. However, clinical studies on inflammatory bowel disease (IBD) patients are lacking. Our goal was to investigate CST concentrations in IBD patients compared to healthy subjects. Additionally, we aimed to determine arterial stiffness parameters in relation to CST. This cross-sectional study compared 80 IBD patients (45 Crohn's disease (CD) and 35 ulcerative colitis (UC) patients) with 75 control subjects. Serum CST levels were significantly higher in the IBD group compared to control subjects $(11.29 \pm 9.14$ vs. $7.13 \pm 6.08 \mathrm{ng} / \mathrm{mL}, p=0.001)$ and in the UC group compared to CD patients $(13.50 \pm 9.58$ vs. $9.03 \pm 6.92 \mathrm{ng} / \mathrm{mL}, p=0.021)$, irrespective of age and BMI. IBD patients exhibited significantly higher values of heart rate adjusted central augmentation index (cAIx-75) (14.88 \pm 10.59 vs. $6.87 \pm 9.50 \%, p<0.001)$ and pulse wave velocity (PWV) $(8.06 \pm 3.23$ vs. $6.42 \pm 1.47 \mathrm{~m} / \mathrm{s}, p<$ 0.001) compared to control group. Furthermore, PWV was the only significant independent correlate of CST ( $B=1.20, t=4.15, p<0.001)$, while CST, PWV, cAIx-75, high-sensitivity C-reactive protein and BMI were significant predictors of positive IBD status (1.089 (1.022-1.161), 1.515 (1.166-1.968), 1.060 (1.024-1.097), 1.458 (1.116-1.906), 0.793 (0.683-0.920), respectively). Serum CST levels were significantly higher in IBD patients compared to controls and an independent positive correlation of CST with PWV existed. Therefore, it is possible that CST could have a role in the complex pathophysiology of IBD and its cardiovascular complications.
\end{abstract}

Keywords: catestatin; inflammatory bowel disease; arterial stiffness

\section{Introduction}

Inflammatory bowel disease (IBD) is a chronic, relapsing disorder that predominately affects small and large intestine, and its two main manifestations are in the form of ulcerative colitis (UC) and Crohn's 
disease (CD) [1]. IBD is believed to be caused by a combination of several predisposing conditions, such as genetic structure, abnormal immunological response and exposure to environmental factors [2]. Furthermore, it is considered that its hallmark characteristic, chronic intestinal inflammation, occurs when an abnormal immunological response causes microvascular endothelial cell damage [3].

Patients with IBD generally have a lower prevalence of classical cardiovascular risk factors, such as obesity, dyslipidemia, and hypertension, but an increased cardiovascular risk in general when compared with healthy population $[4,5]$. The probable reason for this paradox lies in chronic inflammation effect on arteries, and consequently, the heavily connection of IBD with a number of cardiovascular disorders, including atherosclerosis, endothelial dysfunction and increased arterial stiffness [6-8]. In addition, the adverse inflammatory effect on arteries was further confirmed with a recent systematic review by Zanoli et al., that reported a significant increase in pulse wave velocity (PWV), a golden standard for estimation of arterial stiffness, in patients with IBD. Increased arterial stiffness likely presents the initiation and/or progression of atherogenesis and arterial hypertension [9].

Catestatin (CST) is a novel multifunctional peptide proteolytically cleaved from chromogranin A (ChgA), that primarily acts as an inhibitor of catecholamine secretion in vitro in cultured cells and in vivo from mouse adrenal medulla, and as stimulator of histamine release [10-12]. Studies implicate its connection with multiple functions throughout various systems, including vasodilatation [13], immunoregulation [14], insulin resistance [15-17], antimicrobial effect [18,19] and obstructive sleep apnea [20]. Furthermore, it has been reported to be a key regulator of cardiovascular function, with cardioprotective effects including exhibiting the trough suppression of atherosclerosis [21], negative inotropic and lusitropic cardiac effects [22], inhibition of coronary vasoconstriction [21], a reduction in oxidative stress in ischemic-reperfused myocardium [22], a decrease in endothelial inflammation and regulation of blood pressure [10,23-25]. CST is believed to have an important role in the pathophysiology of hypertension, heart failure, reperfusion injury and coronary heart disease [25-27]. Intestinal inflammation has been associated with dysfunctional ChgA production [28]. Consequently, several experimental studies on animal colitis model investigated the relationship of intestinal inflammation and CST secretion [29-32]. Rabbi et al. evaluated the impact of CST on intestinal microbiota in mice and revealed significant changes that could potentially lead to development of new options in treating IBD patients [29]. Furthermore, a few experimental studies have reported that an injection of human CST significantly attenuates intestinal inflammation and severity of inflammatory reactivation through down-regulation of macrophages, pro-inflammatory cytokines and pathways, including interleukin 6 (IL-6), interleukin $1 \beta$, tumor necrosis factor $\alpha$ (TNF- $\alpha$ ) and signal transducer and activator of transcription 3 (STAT3) dependent pathway [30-32]. Muntjewerff et al. even considered CST as an important potential therapeutic modality for various inflammatory and metabolic diseases [33].

Until this point, and to the best of our knowledge, only one study reported serum CST levels in small subset of IBD patients. However, they did not assess any interaction of CST and other clinically relevant factors and biochemical parameters, except C-reactive protein [31]. In addition, there are no published studies that explored the association of CST levels with arterial stiffness parameters, an important surrogate marker of increased cardiovascular risk, in IBD patients. Therefore, the aim of this study was to determine serum CST levels in IBD patients and to investigate its association with arterial stiffness parameters, as well as other biochemical and clinical parameters.

\section{Experimental Section}

\subsection{Study Design}

The study was organized as a cross-sectional observational study. It was conducted at the Department of Gastroenterology, University Hospital of Split, and the Department of Pathophysiology, University of Split School of Medicine over a period from December 2017 to June 2018. 


\subsection{Ethical Considerations}

The study protocol was approved by the Ethics Committee of the University of Split School of Medicine (Class: 003-08/17-03/0001; Registration number: 2181-198-03-04-17-0061; 27 November 2017) and Ethics Committee of the University Hospital of Split (Class: 500-03/17-01/86; Registration number: 2181-147-01/06/M.S.-17-2; 23 November 2017). Written informed consent was obtained from all the study participants prior to the inclusion in the study. The study was conducted in accordance with the statements of the Nuremberg code and ethical principles for medical research as defined by the Declaration of Helsinki and its amendments.

\subsection{Subjects}

This study included 80 patients with IBD, 45 of which had CD and 35 UC. Patients were compared with control group which consisted of 75 healthy subjects. All participants were above 18 years of age and underwent the full study protocol, except fecal calprotectin assessment which was measured only in the patient group. The patients with IBD were recruited from the outpatient clinic of the Department of Gastroenterology, University Hospital of Split. At the beginning of the study, 100 patients were screened for inclusion in the study and 86 patients were found to be eligible for inclusion of the study. Among mentioned, two participants were excluded due to a sudden disease worsening, two due to inability to schedule an appointment and two due to refusal of further procedures (Supplementary Figure S1).

IBD diagnosis was made in accordance with the latest guidelines of the European Crohn's and Colitis Organization and the European Society of Gastrointestinal and Abdominal Radiology [34]. The following inclusion criteria were used: disease duration of at least one year and stable disease activity in the previous 3 months. Exclusion criteria were arterial hypertension; diabetes mellitus and/or use of antidiabetic medication; history of heart failure; history of cardiovascular or cerebrovascular events; known peripheral artery disease; chronic kidney disease; pulmonary disease; liver disease; chronic inflammatory disorders other than IBD; malignant disease and use of local/systemic corticosteroids in the past three months. Control subjects were recruited from the healthy blood donors, acquaintances of the investigators and healthy volunteers from the local primary health care center. All control subjects were screened for the presence of the Rome IV criteria for inflammatory bowel syndrome, as well as any other abdominal symptom suggestive of lactose and gluten intolerance, or any type of gastrointestinal symptoms. If any of these symptoms or conditions were present, we excluded the subjects from the control group.

\subsection{Clinical Assessment and Anthropometric Measurements}

All subjects underwent a detailed physical examination and anthropometric data assessment. A calibrated medical scale with an altitude meter (Seca, Birmingham, UK) was used to measure body mass and height. Body mass index (BMI) was calculated by dividing the value of body mass $(\mathrm{kg})$ by the squared value of height $\left(\mathrm{m}^{2}\right)$. The waist circumference was measured at the level of the midline between the bottom line of the rib arch in the mid-axillary line and the tip of the iliac crests, with the patient standing upright. The hip circumference was measured at the level of the largest circumference of the gluteal muscles, above the line joining the great trochanters of the femur. The waist-hip ratio (WHR) was calculated by dividing waist circumference with the hip circumference value. A standard mercury sphygmomanometer, with a suitable arm, was used to measure arterial blood pressure, and values were obtained after a minimum of two measurements. The subjects were seated, with the upper arm at heart level, and had a 10-min rest period before the measurement. Finally, relevant clinical data were extracted from patients' medical records. Anamnestic data, including tobacco, coffee or alcohol consumption, were taken from all study participants. 


\subsection{Biochemical Analysis}

Blood samples were collected from all participants in the morning period, after at least 10-hour fast. All samples were analyzed in a single laboratory by an experienced biochemist following the same standard procedure. The biochemist was blinded for the participant's assignment to experimental or control group. Blood was drawn from a polyethylene catheter inserted into the antecubital vein. Serum CST (Cat. no. EK-053-27CE, EIA kit, Phoenix Pharmaceuticals Inc., Burlingame, CA, USA) levels were determined by an enzyme-linked immunosorbent assay (ELISA). Manufacturer reported sensitivity of the assay kit for CST of $0.05 \mathrm{ng} / \mathrm{mL}$ with a linear range of $0.05-0.92 \mathrm{ng} / \mathrm{mL}$, and a cross-reactivity with endogenous human CST peptide of $100 \%$ (intra-assay and inter-assay coefficients of variability were $<10 \%$ and $<15 \%$, respectively). Fecal calprotectin concentrations were measured by turbidimetric method (Beckman Coulter AU 680), while plasma high sensitivity C-reactive protein (hs-CRP) levels were determined using a latex turbidimetric method (Abbott Laboratories, Chicago, USA). Other biochemical analyses, including lipid parameter measurements, were measured by standard laboratory methods.

\subsection{Assessment of Disease Severity}

Disease activity in patients with IBD was assessed by two experienced gastroenterology specialists independently, using well-established clinical and endoscopic scoring systems. Where the two differed in scores, a consensus was made. Disease activity among patients with UC was assessed using the UC endoscopic index of severity (UCEIS), Mayo endoscopic score (MES) and Mayo score/disease activity index for UC (Mayo/DAI) [35-37]. Disease severity in patients with Crohn's disease was assessed using simple endoscopic score (SES-CD), the Crohn's disease activity index (CDAI) and the Harvey Bradshaw index (HBI) [37-39]. According to the latest ECCO guidelines, clinical indices should be carefully used since they are not well-validated in the clinical practice and discrepancies could be seen [34]. Therefore, we have stratified our patients using only endoscopic index score, while clinical index scores were only descriptively reported. We have stratified UC and CD group based on the endoscopic disease activity score into different categories. A total of 3 patients have been excluded from the sub-analysis ( 2 from UC and 1 from $C D$ ) due to insufficient number of cases per subgroup.

\subsection{Measurements of Arterial Stiffness}

Applanation tonometry, a widely used standard non-invasive method with a strong correlation to invasively obtained values, has been used for arterial stiffness assessment [39]. Readings were obtained by SphygmoCor (Version 8.1; AtCor Medical, Inc., Sydney, Australia) [40]. Measurements were taken with participants resting in a supine position, in the morning period after an overnight fast, in a temperature-controlled room (constant temperature between $22^{\circ} \mathrm{C}$ and $24{ }^{\circ} \mathrm{C}$ ). Repeated measurements were taken to obtain a minimum of two satisfactory readings according to the built-in quality control requirements. Quality score ranged from zero to 100. Measurements with quality score of less than 90 were repeated in order to acquire acceptable results. Two trained operators performed all vascular measurements. Intra- and inter-operator variability of the PWV measurement were $0.08 \pm$ $0.05 \mathrm{~m} / \mathrm{s}$ and $0.17 \pm 0.10 \mathrm{~m} / \mathrm{s}$, respectively. Readings of the carotid to femoral PWV and pulse wave analysis (PWA) were obtained.

During the measurement of the PWV, a tonometric transducer is placed on the carotid and femoral artery. Distance measurement was taken in a direct line between the points of strongest pulsation of the right carotid/femoral artery and supra-sternal notch, by simple flexible measurement scale device. The intersecting tangent algorithm has been used in the SphygmoCor software and the direct carotid-femoral distance has been corrected for 0.8 to obtain relevant aortic PWV values [40]. The applanation tonometric readings of the carotid and femoral pulse were acquired sequentially in order to do the measurement by a single operator. Device calibration was made based on an average of two consecutive blood pressure measurements. For the assessment of the PWA, a tonometric transducer 
is placed on the subject's radial artery. PWA included peripheral and central augmentation index (pAIx, cAIx), central pulse pressure (cPP), central systolic blood pressure (cSBP), central diastolic blood pressure (cDBP), central mean blood pressure (cMBP), peripheral pulse pressure $(\mathrm{pPP})$, peripheral systolic blood pressure (pSBP), peripheral diastolic blood pressure (pDBP) and peripheral mean blood pressure (pMBP). The values of cAIx were standardized to a resting heart rate of 75 beats-per-minute (cAIx-75) by the SphygmoCor software [41].

\subsection{Statistical Analysis}

Statistical software SPSS (IBM Corp, Armonk, NY, USA; version 25) and SigmaPlot (Systat Software Inc., San Jose, CA, USA; version 14) for Windows was used for statistical data analysis and graph design. Normality of data distribution was first assessed graphically (histograms, Q-Q plots, box plots) and subsequently by the Kolmogorov-Smirnov test. Data were considered normally distributed if all graphical and analytical tests were concordant, whereas any inconsistency was analysed as non-parametric data. Data were expressed as means \pm standard deviation (SD) or median (interquartile range) for continuous variables and as whole numbers and percentages for categorical variables. The Student's t-test, Mann-Whitney U test and Chi-square test were used for the analysis of independent continuous data and categorical data between the IBD and control group, respectively. Fisher's exact test was used for categorical data comparison in the case of small expected value in any cell $(<5)$. Analysis of covariance (ANCOVA) was used to compare group differences in CST and arterial stiffness parameters controlling for important covariates including age and BMI. The aforementioned variables were included in the ANCOVA model due to their relevance to CST levels and arterial stiffness. All the assumptions for ANCOVA analysis were satisfied. Levene's test for the equality of variances was performed prior to the ANCOVA analysis. Moreover, to assess the relationship between different parametric and non-parametric variables Pearson and Spearman correlation methods have been used, respectively. Multiple linear regression analysis with enter selection algorithm was used to determine the relative importance of independent variables (age, BMI, waist circumference, cAIx-75 and PWV) in prediction of CST levels. Finally, predictors of positive IBD status were determined by binomial logistic regression analysis. An odds ratio with a 95\% confidence interval has been used for the presentation of the logistic regression analysis data (OR $(95 \% \mathrm{CI})$ ). The statistical significance was defined as $p<0.05$.

\subsection{Sample Size Analysis}

Sample size analysis has been conducted a priori to the study onset, using a data from a pilot study on 15 healthy subjects and 15 IBD patients. Firstly, graphical estimation has been using nomogram with subsequent confirmation by specific sample size formula. The analysis was calculated using the data on a CST variable. However, sample size of 67 participants per group has been indicated as clinically significant to detect standardized difference of 0.50 , with $80 \%$ power using a cutoff for statistical significance of 0.05 (confidence interval 95\%).

\section{Results}

\subsection{Basic Anthropometric and Related Characteristics of the Study Sample}

There was no significant difference in anthropometric characteristics between studied groups, except in body weight $(75.03 \pm 14.10$ vs. $80.47 \pm 13.28 \mathrm{~kg}, p=0.015)$ and body height $(176.41 \pm 9.27$ vs. $180.03 \pm 9.18 \mathrm{~cm}, p=0.017)$ which were lower in IBD patients. Furthermore, IBD patients had higher prevalence of family history of IBD $(20.0 \%, n=16 \mathrm{vs.} 4.0 \%, n=3, p=0.003)$, while there was no difference in other anamnestic data and habits (Table 1). 
Table 1. Basic characteristics of IBD and control group.

\begin{tabular}{|c|c|c|c|c|}
\hline \multicolumn{2}{|c|}{ Parameter } & $\begin{array}{l}\text { IBD Group } \\
\quad(n=80)\end{array}$ & $\begin{array}{l}\text { Control Group } \\
\quad(n=75)\end{array}$ & $p$ \\
\hline \multicolumn{2}{|c|}{ Age (years) } & $39.79 \pm 14.42$ & $38.47 \pm 12.22$ & $0.543 *$ \\
\hline \multicolumn{2}{|c|}{ Male gender } & $50(62.5 \%)$ & $46(61.3 \%)$ & $0.987^{* *}$ \\
\hline \multicolumn{2}{|c|}{ Body weight (kg) } & $75.03 \pm 14.10$ & $80.47 \pm 13.28$ & $0.015 *$ \\
\hline \multicolumn{2}{|c|}{ Body height $(\mathrm{cm})$} & $176.41 \pm 9.27$ & $180.03 \pm 9.18$ & 0.017 * \\
\hline \multicolumn{2}{|c|}{$\operatorname{BMI}\left(\mathrm{kg} / \mathrm{m}^{2}\right)$} & $24.04 \pm 3.83$ & $24.69 \pm 2.75$ & 0.231 * \\
\hline \multirow{4}{*}{ BMI categories } & $\begin{array}{l}\text { Underweight } \\
\left(<18.5 \mathrm{~kg} / \mathrm{m}^{2}\right)\end{array}$ & $3(3.7 \%)$ & $0(0.0 \%)$ & \multirow{4}{*}{$0.187^{* * *}$} \\
\hline & $\begin{array}{l}\text { Normal weight } \\
\left(18.5-24.9 \mathrm{~kg} / \mathrm{m}^{2}\right)\end{array}$ & $39(48.8 \%)$ & $42(56.0 \%)$ & \\
\hline & $\begin{array}{c}\text { Overweight } \\
\left(25.0-29.9 \mathrm{~kg} / \mathrm{m}^{2}\right)\end{array}$ & $36(45.0 \%)$ & $30(40.0 \%)$ & \\
\hline & $\begin{array}{c}\text { Obesity class I } \\
\left(30.0-34.9 \mathrm{~kg} / \mathrm{m}^{2}\right)\end{array}$ & $2(2.5 \%)$ & $3(4.0 \%)$ & \\
\hline \multicolumn{2}{|c|}{ Waist-hip ratio } & $0.9(0.8-0.9)$ & $0.9(0.8-0.9)$ & $0.172 * * * *$ \\
\hline \multicolumn{2}{|c|}{ Positive family history on CRC } & $13(16.3 \%)$ & $10(13.3 \%)$ & $0.776^{* *}$ \\
\hline \multicolumn{2}{|c|}{ Positive family history on IBD } & $16(20.0 \%)$ & $3(4.0 \%)$ & $0.003^{* * *}$ \\
\hline \multicolumn{2}{|c|}{ Positive family history on CV disease } & $29(36.2 \%)$ & $36(48.0 \%)$ & $0.187^{* *}$ \\
\hline \multicolumn{2}{|c|}{ Alcohol consumption } & $23(28.7 \%)$ & $31(41.3 \%)$ & $0.090 * *$ \\
\hline \multicolumn{2}{|c|}{ Coffee consumption } & $41(51.2 \%)$ & $44(58.7 \%)$ & $0.354^{* *}$ \\
\hline \multicolumn{2}{|c|}{ Active smoking } & $19(23.7 \%)$ & $12(16.4 \%)$ & $0.356^{* *}$ \\
\hline
\end{tabular}

Continuous parametric data are presented as mean \pm standard deviation for parametric data, continuous non-parametric data are presented as median (interquartile range) and categorical data are presented as number (percentage). IBD: inflammatory bowel disease; BMI: body mass index; CRC: colorectal cancer; CV: cardiovascular. * Student $\mathrm{t}$-test for independent samples. ${ }^{* *}$ Chi-square test. ${ }^{* * *}$ Fisher's exact test. ${ }^{* * *}$ Mann-Whitney U test.

Baseline differences in laboratory parameters between the studied groups are shown in Supplementary Table S1.

\subsection{Basic Characteristics of the IBD Group}

There was no significant difference in most anthropometric and disease-related parameters between UC and CD patients. However, CD group did have higher proportion of extraintestinal manifestations $(62.8 \%, n=27$ vs. $18.2 \%, n=6, p<0.001)$ and IBD-related surgical procedures $(35.6 \%$, $n=16$ vs. $2.9 \%, n=1, p<0.001)$.

Furthermore, there was no difference in pharmacologic therapy modalities between IBD subgroups except in aminosalicylates, which were more used in UC patients $(85.7 \%, n=30$ vs. $60.0 \%, n=27$, $p=0.012$.

According to the international endoscopic scoring systems, UC patients had a moderate to severe endoscopic form of disease (UCEIS; MES), while CD patients mostly had moderate endoscopic disease activity (SES-CD) (Table 2). There was no difference in selected variables according to the different disease activity subgroups of UC and CD stratified by endoscopic scores (Supplementary Table S2 and S3).

There was no significant difference in most laboratory parameters between IBD subgroups except in hs-CRP levels which were higher in CD patients (16.43 \pm 12.93 vs. $2.33 \pm 2.19 \mathrm{mg} / \mathrm{L}, p<0.001)$, while UC patients had higher levels of lipid parameters (Supplementary Table S4). 
Table 2. Basic anthropometric and disease characteristics of UC and CD group.

\begin{tabular}{|c|c|c|c|c|}
\hline \multicolumn{2}{|c|}{ Parameter } & $\begin{array}{l}\text { Ulcerative Colitis } \\
\qquad(n=35)\end{array}$ & $\begin{array}{c}\text { Crohn's Disease } \\
\quad(n=45)\end{array}$ & $p$ \\
\hline \multicolumn{2}{|c|}{ Age (years) } & $42.66 \pm 15.57$ & $37.56 \pm 13.38$ & $0.119 *$ \\
\hline \multicolumn{2}{|c|}{ Male gender } & $21(60.0 \%)$ & $29(64.4 \%)$ & $0.684^{* *}$ \\
\hline \multicolumn{2}{|c|}{ Body weight (kg) } & $77.31 \pm 13.03$ & $73.26 \pm 14.92$ & $0.206 *$ \\
\hline \multicolumn{2}{|c|}{ Body height $(\mathrm{cm})$} & $176.29 \pm 8.47$ & $176.51 \pm 10.03$ & $0.915 *$ \\
\hline \multicolumn{2}{|c|}{ BMI $\left(\mathrm{kg} / \mathrm{m}^{2}\right)$} & $24.90 \pm 3.98$ & $23.38 \pm 3.65$ & 0.079 * \\
\hline \multirow{4}{*}{ BMI categories } & $\begin{array}{l}\text { Underweight } \\
\left(<18.5 \mathrm{~kg} / \mathrm{m}^{2}\right)\end{array}$ & $2(5.7 \%)$ & $1(2.2 \%)$ & \\
\hline & $\begin{array}{l}\text { Normal weight } \\
\left(18.5-24.9 \mathrm{~kg} / \mathrm{m}^{2}\right)\end{array}$ & $14(40.0 \%)$ & $25(55.6 \%)$ & $0.093^{* * *}$ \\
\hline & $\begin{array}{c}\text { Overweight } \\
\left(25.0-29.9 \mathrm{~kg} / \mathrm{m}^{2}\right)\end{array}$ & $18(51.4 \%)$ & $18(40.0 \%)$ & \\
\hline & $\begin{array}{c}\text { Obesity class I } \\
\left(30.0-34.9 \mathrm{~kg} / \mathrm{m}^{2}\right)\end{array}$ & $1(2.9 \%)$ & $1(2.2 \%)$ & \\
\hline \multicolumn{2}{|c|}{ Waist-hip ratio } & $0.9(0.8-1.0)$ & $0.9(0.8-0.9)$ & $0.284^{* * * *}$ \\
\hline \multicolumn{2}{|c|}{ SES-CD } & - & $10(5-15)$ & - \\
\hline \multicolumn{2}{|c|}{ CDAI } & - & $57(32-102)$ & - \\
\hline \multicolumn{2}{|c|}{ HBI } & - & $2(1-4)$ & - \\
\hline \multicolumn{2}{|c|}{ UCEIS } & $6(5-7)$ & - & - \\
\hline \multicolumn{2}{|c|}{ MES } & $2(2-3)$ & - & - \\
\hline \multicolumn{2}{|c|}{ Mayo/DAI } & $4(2-7)$ & - & - \\
\hline \multicolumn{2}{|c|}{ Disease duration (years) } & $9(5-11)$ & $6(3-13)$ & $0.268^{* * * *}$ \\
\hline \multicolumn{2}{|c|}{ Positive family history on CRC } & $6(17.1 \%)$ & $7(15.6 \%)$ & $0.849^{* *}$ \\
\hline \multicolumn{2}{|c|}{ Positive family history on IBD } & $7(20.0 \%)$ & $9(20.0 \%)$ & $1.000^{* *}$ \\
\hline \multicolumn{2}{|c|}{ Positive family history on $\mathrm{CV}$ disease } & $11(31.4 \%)$ & $18(40.0 \%)$ & $0.429 * *$ \\
\hline \multicolumn{2}{|c|}{ Alcohol consumption } & $9(25.7 \%)$ & $14(31.1 \%)$ & $0.597^{* *}$ \\
\hline \multicolumn{2}{|c|}{ Active smoking } & $7(20.0 \%)$ & $12(26.7 \%)$ & $0.487^{* *}$ \\
\hline \multicolumn{2}{|c|}{ IBD-related surgical procedures } & $1(2.9 \%)$ & $16(35.6 \%)$ & $<0.001 * * *$ \\
\hline \multicolumn{2}{|c|}{ Extraintestinal manifestations } & $6(18.2 \%)$ & $27(62.8 \%)$ & $<0.001 * *$ \\
\hline \multicolumn{2}{|c|}{ Aminosalicylates } & $30(85.7 \%)$ & $27(60.0 \%)$ & $0.012 * *$ \\
\hline \multicolumn{2}{|c|}{ DMARD } & $12(34.3 \%)$ & $11(24.4 \%)$ & $0.335^{* *}$ \\
\hline \multicolumn{2}{|c|}{ Monoclonal antibodies } & $21(60.0 \%)$ & $30(66.7 \%)$ & $0.538^{* *}$ \\
\hline
\end{tabular}

Continuous parametric data are presented as mean \pm standard deviation, continuous non-parametric data are presented as median (interquartile range) and categorical data are presented as number (percentage). BMI: body mass index; SES-CD: simple endoscopic score for Crohn's disease; CDAI: Crohn's disease activity index; HBI: Harvey-Bradshaw index; UCEIS: ulcerative colitis endoscopic index of severity; MES: Mayo endoscopic score; Mayo/DAI: Mayo score/disease activity index for ulcerative colitis; CRC: colorectal cancer; IBD: inflammatory bowel disease; CV: cardiovascular; DMARD: disease modifying antirheumatic drugs. * Student $t$-test for independent samples. ${ }^{* *}$ Chi-square test. ${ }^{* * *}$ Fisher's exact test. ${ }^{* * * *}$ Mann-Whitney U test.

\subsection{Serum CST Levels}

CST levels were significantly higher in the IBD group in comparison to control subjects $(11.29 \pm$ 9.14 vs. $7.13 \pm 6.08 \mathrm{ng} / \mathrm{mL}, p=0.001$ ). The difference remained significant after adjustment for age and BMI in ANCOVA analysis $(p=0.001)$ (Table 3).

Furthermore, UC group had significantly higher levels of CST in comparison to CD patients $(13.50 \pm 9.58$ vs. $9.03 \pm 6.92 \mathrm{ng} / \mathrm{mL}, p=0.021)$, which remained significant after ANCOVA analysis (Table 3). Moreover, there was no difference in CST levels between IBD subgroups based on median disease duration (Supplementary Table S5). Finally, there was no difference in CST levels between IBD subgroups based on biological therapy $(11.21 \pm 8.22 \mathrm{ng} / \mathrm{mL}$ in biological therapy group vs. $11.42 \pm$ $10.72 \mathrm{ng} / \mathrm{mL}$ in non-biological therapy group, $p=0.923$ ) (Supplementary Table S6). 
Table 3. Comparison of serum CST concentrations between the study groups.

\begin{tabular}{ccccc}
\hline Parameter & \multicolumn{2}{c}{ Study Groups } & \multicolumn{2}{c}{ Test Type } \\
\hline \multirow{3}{*}{ CST $(\mathrm{ng} / \mathrm{mL})$} & IBD $(n=80)$ & Control group $(n=75)$ & $p^{*}$ & $p^{* *}$ \\
\cline { 2 - 5 } & $11.29 \pm 9.14$ & $7.13 \pm 6.08$ & 0.001 & 0.001 \\
& Ulcerative colitis $(n=35)$ & Crohn's disease $(n=45)$ & $p^{*}$ & $p^{* *}$ \\
\cline { 2 - 6 } & $13.50 \pm 9.58$ & $9.03 \pm 6.92$ & 0.021 & 0.036
\end{tabular}

Data are presented as mean \pm standard deviation. CST: catestatin. ${ }^{*}$ Student $\mathrm{t}$-test for independent samples. ** ANCOVA model adjusted for age and BMI.

\subsection{Arterial Stiffness Parameters}

IBD patients exhibited statistically significantly higher values of cAIx (16.36 \pm 9.95 vs. $10.31 \pm$ $8.19 \%, p<0.001)$, cAIx-75 (14.88 \pm 10.59 vs. $6.87 \pm 9.50 \%, p<0.001)$, PWV $(8.06 \pm 3.23$ vs. $6.42 \pm$ $1.47 \mathrm{~m} / \mathrm{s}, p<0.001)$ and heart rate $(72.04 \pm 12.21 \mathrm{vs} .68 .13 \pm 9.14 \mathrm{bpm}, p=0.027)$ in comparison to control group, while there was no difference in other parameters of arterial stiffness. Importantly, differences in all the aforementioned parameters were still significant after adjustment for age and BMI $(p<0.05)$. Furthermore, IBD group had significantly higher proportion of subjects with end-organ damage marked by PWV $>10 \mathrm{~m} / \mathrm{s}(17.5 \%, n=14$ vs. $2.7 \%, n=1, p=0.002)$ (Table 4) [40]. This pattern persisted in analysis using specific age-adjusted PWV reference values $(13.8 \%, n=11$ in IBD group vs. $0.0 \%, n=0$ in control group) (Supplementary Table S7) [42]. Furthermore, participant group with positive PWV criteria for end-organ damage (PWV $>10 \mathrm{~m} / \mathrm{s}$ ) had significantly higher CST levels (20, 7-23 vs. 7, 4-11 ng/mL, $p=0.001$ ) (Supplementary Table S5).

Table 4. Comparison of selected arterial stiffness parameters between IBD and control group.

\begin{tabular}{ccccc}
\hline Parameter & $\begin{array}{c}\text { IBD Group } \\
(\boldsymbol{n}=\mathbf{8 0})\end{array}$ & $\begin{array}{c}\text { Control Group } \\
(\boldsymbol{n}=\mathbf{7 5})\end{array}$ & $\boldsymbol{p}^{*}$ & $\boldsymbol{p}^{* *}$ \\
\hline pSBP $(\mathrm{mmHg})$ & $120.63 \pm 7.15$ & $118.57 \pm 7.32$ & 0.080 & 0.058 \\
pDBP (mmHg) & $74.44 \pm 6.71$ & $73.39 \pm 9.16$ & 0.419 & 0.483 \\
pMBP (mmHg) & $89.78 \pm 5.98$ & $88.44 \pm 6.96$ & 0.201 & 0.194 \\
pPP (mmHg) & $46.19 \pm 7.17$ & $45.19 \pm 10.67$ & 0.497 & 0.333 \\
cSBP (mmHg) & $106.13 \pm 6.97$ & $104.37 \pm 6.43$ & 0.106 & 0.087 \\
cDBP (mmHg) & $75.29 \pm 6.54$ & $74.16 \pm 6.49$ & 0.283 & 0.264 \\
cMBP (mmHg) & $85.55 \pm 5.99$ & $84.24 \pm 6.17$ & 0.182 & 0.154 \\
cPP (mmHg) & $30.84 \pm 6.27$ & $30.21 \pm 3.98$ & 0.458 & 0.420 \\
HR (bpm) & $72.04 \pm 12.21$ & $68.13 \pm 9.14$ & 0.027 & 0.037 \\
pAIx (\%) & $-37.30 \pm 16.13$ & $-40.41 \pm 15.48$ & 0.223 & 0.529 \\
cAIx (\%) & $16.36 \pm 9.95$ & $10.31 \pm 8.19$ & $<0.001$ & 0.005 \\
cAIx-75 (\%) & $14.88 \pm 10.59$ & $6.87 \pm 9.50$ & $<0.001$ & 0.003 \\
PWV (m/s) & $8.06 \pm 3.23$ & $6.42 \pm 1.47$ & $<0.001$ & $<0.001$ \\
End-organ damage ${ }^{\text {a }}$ & $14(17.5 \%)$ & $2(2.7 \%)$ & $0.002 * *$ & n/a \\
\hline
\end{tabular}

Continuous data are presented as mean \pm standard deviation and categorical data are presented as number (percentage). pSBP: peripheral systolic blood pressure; pDBP: peripheral diastolic blood pressure; pMBP: peripheral mean blood pressure; pPP: peripheral pulse pressure; CSBP: central systolic blood pressure; cDBP: central diastolic blood pressure; cMBP: central mean blood pressure; $\mathrm{CPP}$ : central pulse pressure; HR: heart rate; bpm: beats per minute; pAIx: peripheral augmentation index; cAIx: central augmentation index; cAIx-75: central augmentation index corrected for heart rate; PWV: pulse wave velocity. ${ }^{*}$ Student $t$-test for independent samples. ${ }^{* *}$ ANCOVA model adjusted for age and BMI. ${ }^{* * *}$ Chi-square test. ${ }^{\text {a }}$ According to the ESC/EHA guidelines, $\mathrm{PWV}>10 \mathrm{~m} / \mathrm{s}$ is considered to represent an end-organ damage [40].

Finally, there was no significant difference in arterial stiffness parameters between UC and CD (Supplementary Table S8). Moreover, there was no difference in arterial stiffness parameters between IBD subgroups based on biological therapy $(p>0.05)$ (Supplementary Table S6). 


\subsection{Bivariate Correlation Analysis of CST and Selected Parameters}

CST exhibited statistically significant correlation with age $(r=0.18, p=0.023)$, cAIx-75 $(r=0.19$, $p=0.003), \operatorname{PWV}(r=0.37, p<0.001)$ and disease duration $(r=-0.24, p=0.032)$ while there was no significant correlation between CST and other variables (Table 5). There was no significant association of disease duration and PWV, but WBC positively correlated with PWV $(r=0.24, p=0.031$ and $r=0.12$, $p=0.352$, respectively).

Table 5. Correlation between parameters of CTS and selected parameters.

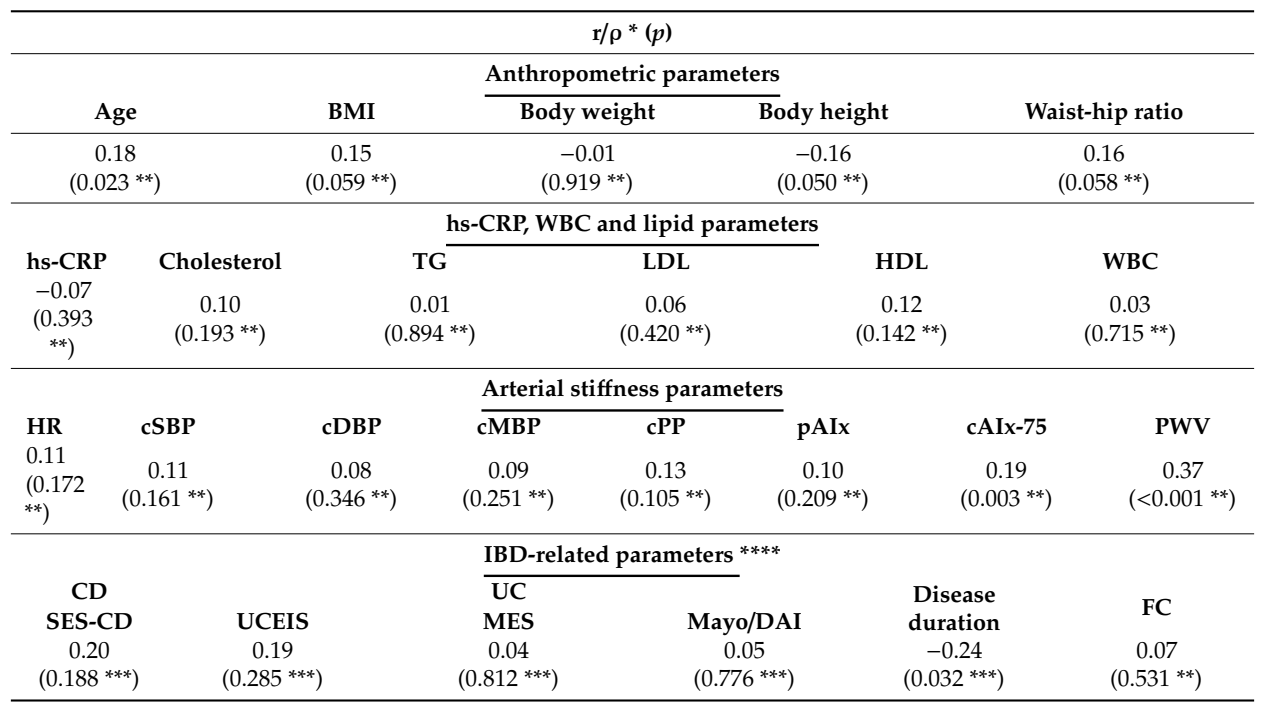

BMI: body mass index; hs-CRP: high sensitivity C-reactive protein; TG: triglycerides; LDL: low density lipoproteins; HDL: high density lipoproteins; WBC: white blood cell; HR: heart rate; cSBP: central systolic blood pressure; cDBP: central diastolic blood pressure; cMBP: central mean blood pressure; cPP: central pulse pressure; pAIx: peripheral augmentation index; cAIx-75: central augmentation index corrected for heart rate; PWV: pulse wave velocity; SES-CD: simple endoscopic score for Crohn's disease; UCEIS: ulcerative colitis endoscopic index of severity; MES: Mayo endoscopic score; Mayo/DAI: Mayo score/disease activity index for ulcerative colitis; FC: fecal calprotectin. ${ }^{*} \mathrm{r} / \rho$-value indicates correlation coefficient. ${ }^{* *}$ Pearson's correlation test. ${ }^{* * *}$ Spearman's correlation test. ${ }^{* * *}$ Correlation in the IBD group.

\subsection{Multiple Linear Regression Analysis of Selected Parameters as Independent Predictors of CST}

The multiple linear regression model showed that PWV was the only independent predictor of CST levels $(B=1.20, p<0.001)$. Other variables included in the model did not reach statistical significance (Table 6).

Table 6. Multiple linear regression analysis of selected variables as independent predictors of CST.

\begin{tabular}{ccc}
\hline Parameter & $\boldsymbol{B}^{*}\left(\boldsymbol{t}^{* *}\right) \boldsymbol{p}$ & Overall \\
\hline Age & $-0.07(-1.11) 0.270$ & \\
BMI & $0.10(0.50) 0.620$ & $R^{2}$ adjusted $=0.133$ \\
Waist-hip ratio & $0.36(1.32) 0.189$ & F ratio $=5.739$ \\
cAIx-75 & $0.07(1.68) 0.095$ & $p<0.001$ \\
PWV & $1.20(4.15)<0.001$ &
\end{tabular}

BMI: body mass index; cAIx-75: central augmentation index corrected for heart rate; PWV: pulse wave velocity. * $\mathrm{B}$-value indicates regression coefficient of the independent variable. ${ }^{* *} \mathrm{t}$-value indicates $\mathrm{t}$-statistic value.

\subsection{Odds for Selected Variables in Prediction of the IBD Status}

Binomial logistic regression revealed that CST (1.089 (1.022-1.161), $p=0.009)$, PWV (1.515 (1.166-1.968), $p=0.002)$, cAIx-75 (1.060 (1.024-1.097), $p=0.001)$, hs-CRP (1.458 (1.116-1.906), $p=0.006)$ and BMI $(0.793(0.683-0.920), p=0.002)$ were significant predictors of positive IBD status (Table 7). 
Table 7. Binomial logistic regression for the prediction of IBD status.

\begin{tabular}{ccc}
\hline Predictor & OR $(\mathbf{9 5} \% \mathbf{C I})$ & $p$ \\
\hline CST & $1.089(1.022-1.161)$ & 0.009 \\
BMI & $0.793(0.683-0.920)$ & 0.002 \\
PWV & $1.515(1.166-1.968)$ & 0.002 \\
cAIx-75 & $1.060(1.024-1.097)$ & 0.001 \\
hs-CRP & $1.458(1.116-1.906)$ & 0.006 \\
\hline
\end{tabular}

CST: catestatin; BMI: body mass index; PWV: pulse wave velocity; cAIx-75: central augmentation index corrected for heart rate; hs-CRP: high sensitivity C-reactive protein.

\section{Discussion}

This study has shown that IBD patients had significantly higher levels of serum CST and arterial stiffness parameters in comparison to healthy subjects. Correspondingly, CST and PWV proved to be independent predictors of positive IBD status. In addition, an independent positive correlation exists between CST and PWV which could emphasize the relationship between IBD, CST and increased arterial stiffness. However, the role of CST in the pathophysiology of IBD and a complex link between IBD and increased cardiovascular risk have not been well-established $[9,14,43]$.

To the best of our knowledge, only one study thus far has measured serum CST levels in a small sample of IBD patients. However, serum CST concentrations were not their primary outcome and other relevant clinical information, as well as biochemical parameters are lacking in that study [31]. Therefore, we believe that this study is the first clinical study to investigate serum CST levels in the IBD population and control subjects with detailed relevance to disease characteristics. In addition, this is the first study to analyze the relationship of CST and arterial stiffness parameters in the IBD population.

It has been previously shown that inflammatory process affects the secretion of enterochromaffine cells in the gut of IBD patients [20]. Accordingly, it seems that ChgA, a precursor of CST, is increased in colon and plasma in patients with IBD [31,44,45].

Derivatives of ChgA have been also connected to the pathophysiology of gastrointestinal diseases. Importantly, animal studies have shown that CST levels are significantly increased in experimental colitis model. In fact, it seems that ChgA and CST expression increases simultaneously during the iatrogenous development of collitis [31].

Findings of this study indicate that IBD patients have increased serum levels of CST in comparison to healthy subjects. Additionally, we have found that CST is an independent predictor of the IBD status. Similar to this study, Rabbi et al. found significantly higher levels of serum CST in a small sample of IBD patients [31]. However, they did not establish any difference in CST and ChgA levels between the different IBD subgroups, whereas we have found significantly higher concentrations of serum CST in UC patients [31]. Based on the existing literature, it is hard to detect reasons for this discrepancy, but it is possible that these differences in CST levels between UC and CD could be accounted for disparity in different disease activity. Specifically, UC patients had higher disease activity compared to the CD group in the present study. On contrary, study by Eissa et al. showed significantly decreased expression of CST in colonic biopsy specimens of the UC patients compared to control subjects [32]. Additionally, there was no difference in CST, PWV and cAIx-75 between the different disease activity subgroups of UC and CD. However, these results should be interpreted with caution, due to the relatively small number of cases in each subgroup. Therefore, further studies are necessary in order to confirm the role of CST in the pathophysiology of UC and CD.

Previous studies on animal models have demonstrated that CST could have a beneficial therapeutic role in IBD [29-32]. Specifically, experimental animal studies have shown that CST could have a modulatory role in inflammatory response. It has been suggested that CST could attenuate the intensity of inflammation in IBD and even possibly prevent relapse of the disease [30]. Consistently, CST delayed the onset of induced-colitis in murine models in a dose-dependent way [31,32]. Furthermore, it has been shown that the intrarectal administration of CST improves stool consistency and decreases hematochezia 
in murine colitis models [32]. CST treatment also significantly reduced disease severity with improved macroscopic and histologic disease scores [31,32]. These effects have been mainly mediated by local effects such as decreased colonic mucosal inflammation, edema and cellular infiltration. Similarly, we did not establish any significant correlation between CST and body weight/BMI in this study.

Several mechanisms have been proposed for these positive effects of CST on gut media in IBD. Primarily, CST induces a decrease in colonic M1 macrophages, local concentrations of proinflammatory cytokines (IL-1 $\beta$, IL-6, TNF- $\alpha$ ), activity of myeloperoxidase and CRP in animal models [30,31]. Moreover, colonic expression of CST was negatively correlated with proinflammatory cytokines interleukin 8 (IL-8) and interleukin 18 (IL-18), while CST therapy reduced IL-18 levels in UC patients [32]. Interestingly, in vivo and in vitro studies have shown that CST treatment only decreased proinflammatory cytokine and macrophage levels without any effect on anti-inflammatory cytokines and M2 macrophages [30]. Aforementioned findings could be the reason why CST treatment did not have any effect on gut histology and cytokine levels in animal models without colitis [31]. A possible mediator in this anti-inflammatory action is STAT3, a well-known factor in the pathophysiology of IBD [46]. Mouse models who were treated with high-dose CST exhibited significantly higher levels of phosphorylated STAT3 in the colon, but absolute STAT3 levels were not affected $[24,25]$. Importantly, when STAT3 blocker was added, cytokine secretion from the macrophages in experimental colitis was not decreased by CST [31,32]. Furthermore, it has been shown that CST therapy maintains steady levels of tight junction proteins which are a crucial indicators of cell membrane homeostasis in the gut [32,47]. Additionally, studies have shown that CST has the ability to influence the chemotaxis of monocytes [48]. Therefore, it appears that CST could yield immunomodulatory effects in the gut; however, further studies are necessary $[49,50]$.

Several studies have shown that IBD patients have a higher cardiovascular risk in comparison to the general population, even though they have a lower prevalence of traditional cardiovascular risk factors $[4,5,51]$. This has been confirmed in this study by obtaining increased arterial stiffness and lower values of lipid parameters in IBD subjects. Arterial stiffness is a well-established predictor of the cardiovascular mortality and morbidity [42]. Findings of increased arterial stiffness in IBD patients compared to healthy subjects are consistent with most previous studies [9,52]. A pioneer study in 2012 on IBD patients showed increased arterial stiffness in comparison to healthy subjects [53]. Importantly, meta-analysis by Zanoli et al. confirmed significantly increased carotid-femoral PWV in IBD patients [9]. Consequently, IBD patients in the present study had significantly higher prevalence of end-organ damage detected by PWV $>10 \mathrm{~m} / \mathrm{s}$.

Complex pathophysiology responsible for this paradoxical association of IBD and cardiovascular risk has not been well-determined. CST has been implicated in the pathophysiology of cardiovascular diseases and studies suggest that it has vasodilatative and antihypretensive properties [11]. Furthermore, anti-atherosclerotic, antihypertensive and cardioprotective effects have been demonstrated in previous studies $[10,16,19]$. Therefore, it could be possible that CST has a mediatory role in the interconnection of IBD and cardiovascular events. Results of the present study show significant negative correlation of disease duration and CST. However, subgroup comparison based on median disease duration did not show significant difference in CST. Taking into account the relatively small number of patients, future cohort longitudinal studies are necessary to elucidate the real association of disease duration and CST levels. The present findings indicate that PWV, the most important marker of arterial stiffness, is independently positively associated with serum CST levels. Importantly, PWV was the only independent predictor of CST levels. Consistently, subgroup of subjects with markers of end-organ damage have significantly higher levels of CST. Supporting findings were reported in a study on the general Japanese population, which found positive association of CST and PWV [43]. Considering that endothelial dysfunction, one of the key mechanisms for increased arterial stiffness, is largely precipitated by systemic inflammation, and that CST has speculated immunomodulatory roles, it is possible that CST could have a role in the interconnection of IBD and cardiovascular diseases [3]. From another aspect, sympathetic nervous system (SNS) activity might be an important contributor 
to pathologic arterial remodeling and this might translate to increased arterial stiffness parameters as postulated in previous clinical studies [54-56]. Importantly, sympathetic overactivity is present in patients with ulcerative colitis [57,58]. Since CST is an important antiadrenergic peptide secreted peripherally in a compensatory fashion to counterbalance catecholamine surge, elevated CST levels might simply reflect increased SNS activation and CST might be the surrogate marker of this activity. This might partially explain the high circulating CST levels in this population along with the observed increased parameters of arterial stiffness. Of note, CST and other peptides that are derived from ChgA are co-stored and co-released together with catecholamines from the storage vesicles of adrenal chromaffin cells and adrenergic neurons while circulating norepinephrine levels paralleled CST levels in a concentration-dependent manner [59]. Since the physiological effects of norepinephrine and SNS mediators promote tachyphylaxis and systemic vasoconstriction it is possible that these effects contributed to detrimental vascular remodeling as evident in increased arterial stiffness parameters. Finally, although CST stand-alone generally confers protective effects on heart and vasculature, as shown in a handful of previous studies $[22,23,60,61]$, it is possible that in the setting of IBD, a disease state characterized by persistent inflammation and increased SNS activity, effects of CST, despite being elevated in circulation, are not sufficient to compensate for adverse arterial remodeling that is propagated by the proinflammatory and adrenergic cellular pathways. However, due to a substantial evidence gap in this regard and lack of studies reporting on CST and arterial stiffness parameters, these hypothetical interactions need to be confirmed in future preclinical and mechanistic studies.

We did not establish any difference in arterial stiffness parameters between IBD subgroups, which is consistent with the meta-analysis by Zanoli et al. and pilot research on the pediatric IBD population [9,62]. Furthermore, there was no difference in CST and arterial stiffness between different groups according to the anti-TNF- $\alpha$ therapy in the present study. However, there is evidence of lower PWV in IBD patients on anti-TNF- $\alpha$ therapy [9]. Finally, consistent with this study, the meta-analysis by Zanoli et al. did not find any difference in PWV between IBD patients with active disease and remission [9].

Interesting findings were reported by Zanoli et al. in a recently published individual participant data meta-analysis, indicating that PWV was associated with disease duration and white blood cell count [63]. On contrary, the present research did not reveal any association between disease duration and PWV but showed significant positive correlation of white blood cell and PWV which is in accordance to previously mentioned findings. These results confirm the important interaction of systemic inflammation and arterial stiffness in IBD patients.

This study has several limitations. First, the design of the present study was cross-sectional, which affects the establishment of true causal relationship. Second, this study was conducted as a single-center study and had a relatively small number of participants. The IBD group from our study mostly was heterogenous in disease activity which additionally decreases the sample size by stratification and impedes the generalization of the findings to the entire IBD population. Moreover, as IBD is relapsing and remitting disease, secretion pattern of biomarkers is not constant and therefore one single measurement cannot be an ideal strategy for determination of causal relationship. Furthermore, any time dependent effects of IBD cannot be truly observed by our study model. Additionally, we did not measure all inflammatory parameters which could have an important role in clarifying some of the results, except hs-CRP. Finally, direct markers of sympathetic nervous system activity, such as circulating catecholamines or urinary catecholamines, were not measured in this study due to financial constraints and thus they are only indirectly discussed.

\section{Conclusions}

In conclusion, serum CST levels and arterial stiffness parameters are significantly increased in IBD patients compared to healthy subjects, which could indicate a higher cardiovascular risk in this population. However, future observational studies are necessary to explain complex pathophysiology of CST and arterial stiffness in the IBD population. 
Supplementary Materials: The following are available online at http://www.mdpi.com/2077-0383/9/3/628/s1, Figure S1: Flow diagram, Table S1: Detailed laboratory characteristics of IBD and control group, Table S2: Detailed laboratory characteristics of UC and CD group, Table S3: Comparison of CST levels between IBD subgroups according to median of disease duration and PWV thresholds for end-organ damage, Table S4: Comparison of CST levels between IBD subgroups according to biologic therapy, Table S5: Prevalence of abnormal age-adjusted PWV valuess among studied groups, Table S6: Comparison of selected arterial stiffness parameters between UC and CD subgroup.

Author Contributions: Conceptualization, P.M.Z., A.M., I.T.H., D.R., M.V., D.S.-D., J.A.B., I.M., A.T. and J.B.; Data curation, P.M.Z., A.M., I.T.H. and J.B.; Formal analysis, P.M.Z., A.M. and J.B.; Investigation, P.M.Z., A.M., I.T.H. and J.B.; Methodology, P.M.Z., I.T.H., D.R., M.V., D.S.-D., J.A.B., I.M. and A.T.; Project administration, A.T. and J.B.; Resources, P.M.Z., A.M., D.R. and J.B.; Supervision, P.M.Z., A.T. and J.B.; Validation, P.M.Z., D.S.-D., J.A.B. and J.B.; Visualization, P.M.Z., A.M. and J.B.; Writing—original draft, P.M.Z., A.M. and J.B.; Writing-review and editing, P.M.Z., A.M., I.T.H., D.R., M.V., D.S.-D., J.A.B., I.M., A.T. and J.B. All authors have read and agreed to the published version of the manuscript.

Funding: This research received no external funding.

Acknowledgments: We would like to express strong gratitude to Mladen Boban from the Department of Pharmacology, University of Split School of Medicine, for providing the laboratory equipment and facilities for the study conduction.

Conflicts of Interest: The authors declare no conflict of interest.

\section{References}

1. de Souza, H.S.P. Etiopathogenesis of inflammatory bowel disease: Today and tomorrow. Curr. Opin. Gastroenterol. 2017, 33, 222-229. [CrossRef] [PubMed]

2. Ribaldone, D.; Pellicano, R.; Actis, G. Pathogenesis of inflammatory bowel disease: Basic science in the light of real-world epidemiology. Gastrointest. Disord. 2018, 1, 10. [CrossRef]

3. Hatoum, O.A.; Binion, D.G. The vasculature and inflammatory bowel disease: Contribution to pathogenesis and clinical pathology. Inflamm. Bowel Dis. 2005, 11, 304-313. [CrossRef] [PubMed]

4. Harbord, M.; Annese, V.; Vavricka, S.R.; Allez, M.; Barreiro-de Acosta, M.; Boberg, K.M.; Burisch, J.; De Vos, M.; De Vries, A.M.; Dick, A.D.; et al. The first European evidence-based consensus on extra-intestinal manifestations in inflammatory bowel disease. J. Crohns Colitis 2016, 10, 239-254. [CrossRef] [PubMed]

5. Yarur, A.J.; Deshpande, A.R.; Pechman, D.M.; Tamariz, L.; Abreu, M.T.; Sussman, D.A. Inflammatory bowel disease is associated with an increased incidence of cardiovascular events. Am. J. Gastroenterol. 2011, 106, 741-747. [CrossRef]

6. Cappello, M.; Licata, A.; Calvaruso, V.; Bravatà, I.; Aiello, A.; Torres, D.; Della Corte, V.; Tuttolomondo, A.; Perticone, M.; Licata, G.; et al. Increased expression of markers of early atherosclerosis in patients with inflammatory bowel disease. Eur. J. Intern. Med. 2017, 37, 83-89. [CrossRef]

7. Principi, M.; Mastrolonardo, M.; Scicchitano, P.; Gesualdo, M.; Sassara, M.; Guida, P.; Bucci, A.; Zito, A.; Caputo, P.; Albano, F.; et al. Endothelial function and cardiovascular risk in active inflammatory bowel diseases. J. Crohns Colitis 2013, 7, e427-e433. [CrossRef]

8. Korkmaz, H.; Sahin, F.; Ipekci, S.H.; Temel, T.; Kebapcilar, L. Increased pulse wave velocity and relationship with inflammation, insulin, and insulin resistance in inflammatory bowel disease. Eur. J. Gastroenterol. Hepatol. 2014, 26, 725-732. [CrossRef]

9. Zanoli, L.; Rastelli, S.; Granata, A.; Inserra, G.; Empana, J.P.; Boutouyrie, P.; Laurent, S.; Castellino, P. Arterial stiffness in inflammatory bowel disease: A systematic review and meta-analysis. J. Hypertens. 2016, 34, 822-829. [CrossRef]

10. Mahata, S.K.; Mahata, M.; Fung, M.M.; O'Connor, D.T. Catestatin: A multifunctional peptide from chromogranin A. Regul. Pept. 2010, 162, 33-43. [CrossRef]

11. Mahata, S.K.; O'Connor, D.T.; Mahata, M.; Yoo, S.H.; Taupenot, L.; Wu, H.; Gill, B.M.; Parmer, R.J. Novel autocrine feedback control of catecholamine release. A discrete chromogranin a fragment is a noncompetitive nicotinic cholinergic antagonist. J. Clin. Investig. 1997, 100, 1623-1633. [CrossRef] [PubMed]

12. Livett, B.G.; Boksa, P.; Dean, D.M.; Mizobe, F.; Lindenbaum, M.H. Use of isolated chromaffin cells to study basic release mechanisms. J. Auton. Nerv. Syst. 1983, 7, 59-86. [CrossRef] 
13. Fung, M.M.; Salem, R.M.; Mehtani, P.; Thomas, B.; Lu, C.F.; Perez, B.; Rao, F.; Stridsberg, M.; Ziegler, M.G.; Mahata, S.K.; et al. Direct vasoactive effects of the chromogranin A (CHGA) peptide catestatin in humans in vivo. Clin. Exp. Hypertens. 2010, 32, 278-287. [CrossRef] [PubMed]

14. Aung, G.; Niyonsaba, F.; Ushio, H.; Kajiwara, N.; Saito, H.; Ikeda, S.; Ogawa, H.; Okumura, K. Catestatin, a neuroendocrine antimicrobial peptide, induces human mast cell migration, degranulation and production of cytokines and chemokines. Immunology 2011, 132, 527-539. [CrossRef]

15. Simunovic, M.; Supe-Domic, D.; Karin, Z.; Degoricija, M.; Paradzik, M.; Bozic, J.; Unic, I.; Skrabic, V. Serum catestatin concentrations are decreased in obese children and adolescents. Pediatr. Diabetes 2019, 20, 549-555. [CrossRef]

16. Ying, W.; Mahata, S.; Bandyopadhyay, G.K.; Zhou, Z.; Wollam, J.; Vu, J.; Mayoral, R.; Chi, N.W.; Webster, N.; Corti, A.; et al. Catestatin inhibits obesity-induced macrophage infiltration and inflammation in the liver and suppresses hepatic glucose production, leading to improved insulin sensitivity. Diabetes 2018, 67, 841-848. [CrossRef]

17. Bandyopadhyay, G.; Vu, C.; Gentile, S.; Lee, H.; Biswas, N.; Chi, N.W.; O'Connor, D.T.; Mahata, S.K. Catestatin (Chromogranin A352-372) and novel effects on mobilization of fat from adipose tissue through regulation of adrenergic and leptin signaling. J. Biol. Chem. 2012, 287, 23141-23151. [CrossRef]

18. Radek, K.A.; Lopez-Garcia, B.; Hupe, M.; Niesman, I.R.; Elias, P.M.; Taupenot, L.; Mahata, S.K.; O'Connor, D.T.; Gallo, R.L. The neuroendocrine peptide catestatin is a cutaneous antimicrobial and induced in the skin after injury. J. Investig. Dermatol. 2008, 128, 1525-1534. [CrossRef]

19. Briolat, J.; Wu, S.D.; Mahata, S.K.; Gonthier, B.; Bagnard, D.; Chasserot-Golaz, S.; Helle, K.B.; Aunis, D.; Metz-Boutigue, M.H. New antimicrobial activity for the catecholamine release-inhibitory peptide from chromogranin A. Cell Mol. Life Sci. 2005, 62, 377-385. [CrossRef]

20. Borovac, J.; Dogas, Z.; Supe-Domic, D.; Galic, T.; Bozic, J. Catestatin serum levels are increased in male patients with obstructive sleep apnea. Sleep Breath 2018, 23, 473-481. [CrossRef]

21. Kojima, M.; Ozawa, N.; Mori, Y.; Takahashi, Y.; Watanabe-Kominato, K.; Shirai, R.; Watanabe, R.; Sato, K.; Matsuyama, T.A.; Ishibashi-Ueda, H.; et al. Catestatin prevents macrophage-driven atherosclerosis but not arterial injury-induced neointimal hyperplasia. Thromb. Haemost. 2018, 118, 182-194. [CrossRef] [PubMed]

22. Chu, S.Y.; Peng, F.; Wang, J.; Liu, L.; Meng, L.; Zhao, J.; Xiao-Ning, H.; Wen-Hui, D. Catestatin in defense of oxidative-stress-induced apoptosis: A novel mechanism by activating the beta2 adrenergic receptor and $\mathrm{PKB} /$ Akt pathway in ischemic-reperfused myocardium. Peptides 2020, 123, 170200. [CrossRef] [PubMed]

23. Chen, H.; Liu, D.; Ge, L.; Wang, T.; Ma, Z.; Han, Y.; Duan, Y.; Xu, X.; Liu, W.; Yuan, J.; et al. Catestatin prevents endothelial inflammation and promotes thrombus resolution in acute pulmonary embolism in mice. Biosci. Rep. 2019, 39, BSR20192236. [CrossRef] [PubMed]

24. Angelone, T.; Quintieri, A.M.; Brar, B.K.; Limchaiyawat, P.T.; Tota, B.; Mahata, S.K.; Cerra, M.C. The antihypertensive chromogranin a peptide catestatin acts as a novel endocrine/paracrine modulator of cardiac inotropism and lusitropism. Endocrinology 2008, 149, 4780-4793. [CrossRef] [PubMed]

25. Mahapatra, N.R. Catestatin is a novel endogenous peptide that regulates cardiac function and blood pressure. Cardiovasc. Res. 2008, 80, 330-338. [CrossRef] [PubMed]

26. Borovac, J.; Glavas, D.; Susilovic Grabovac, Z.; Supe Domic, D.; D'Amario, D.; Bozic, J. Catestatin in acutely decompensated heart failure patients: insights from the CATSTAT-HF study. J. Clin. Med. 2019, 8, 1132. [CrossRef]

27. Zhao, Y.; Zhu, D. Potential applications of catestatin in cardiovascular diseases. Biomark. Med. 2016, 10, 877-888. [CrossRef]

28. Mahata, S.K.; Kiranmayi, M.; Mahapatra, N.R. Catestatin: A master regulator of cardiovascular functions. Curr. Med. Chem. 2018, 25, 1352-1374. [CrossRef]

29. El-Salhy, M.; Danielsson, A.; Stenling, R.; Grimelius, L. Colonic endocrine cells in inflammatory bowel disease. J. Intern. Med. 1997, 242, 413-419. [CrossRef]

30. Rabbi, M.F.; Munyaka, P.M.; Eissa, N.; Metz-Boutigue, M.H.; Khafipour, E.; Ghia, J.E. Human catestatin alters gut microbiota composition in mice. Front. Microbiol. 2017, 7, 2151. [CrossRef]

31. Rabbi, M.F.; Eissa, N.; Munyaka, P.M.; Kermarrec, L.; Elgazzar, O.; Khafipour, E.; Bernstein, C.N.; Ghia, J.E. Reactivation of intestinal inflammation is suppressed by catestatin in a murine model of colitis via $\mathrm{m} 1$ macrophages and not the gut microbiota. Front. Immunol. 2017, 8, 985. [CrossRef] 
32. Rabbi, M.F.; Labis, B.; Metz-Boutigue, M.H.; Bernstein, C.N.; Ghia, J.E. Catestatin decreases macrophage function in two mouse models of experimental colitis. Biochem. Pharmacol. 2014, 89, 386-398. [CrossRef] [PubMed]

33. Eissa, N.; Hussein, H.; Mesgna, R.; Bonin, S.; Hendy, G.N.; Metz-Boutigue, M.H.; Bernstein, C.N.; Ghia, J.E. Catestatin regulates epithelial cell dynamics to improve intestinal inflammation. Vaccines 2018, 6, 67. [CrossRef] [PubMed]

34. Muntjewerff, E.M.; Dunkel, G.; Nicolasen, M.J.T.; Mahata, S.K.; van den Bogaart, G. Catestatin as a target for treatment of inflammatory diseases. Front. Immunol. 2018, 9, 2199. [CrossRef] [PubMed]

35. Maaser, C.; Sturm, A.; Vavricka, S.R.; Kucharzik, T.; Fiorino, G.; Annese, V.; Calabrese, E.; Baumgart, D.C.; Bettenworth, D.; Borralho Nunes, P.; et al. ECCO-ESGAR Guideline for Diagnostic Assessment in IBD Part 1: Initial diagnosis, monitoring of known IBD, detection of complications. J. Crohn Colitis 2019, 13, 144-164K. [CrossRef] [PubMed]

36. Travis, S.; Schnell, D.; Krzeski, P.; Abreu, M.T.; Altman, D.G.; Colombel, J.F.; Feagan, B.G.; Hanauer, S.B.; Lichtenstein, G.R.; Marteau, P.R.; et al. Reliability and initial validation of the ulcerative colitis endoscopic index of severity. Gastroenterology 2013, 145, 987-995. [CrossRef]

37. Xie, T.; Zhang, T.; Ding, C.; Dai, X.; Li, Y.; Guo, Z.; Wei, Y.; Gong, J.; Zhu, W.; Li, J. Ulcerative colitis endoscopic index of severity (UCEIS) versus Mayo endoscopic score (MES) in guiding the need for colectomy in patients with acute severe colitis. Gastroenterol. Rep. 2017, 6, 38-44. [CrossRef]

38. Lewis, J.; Chuai, S.; Nessel, L.; Lichtenstein, G.R.; Aberra, F.N.; Ellenberg, J.H. Use of the noninvasive components of the mayo score to assess clinical response in ulcerative colitis. Inflamm. Bowel Dis. 2008, 14, 1660-1666. [CrossRef]

39. Daperno, M.; D’Haens, G.; van Assche, G.; Baert, F.; Bulois, P.; Maunoury, V.; Sostegni, R.; Rocca, R.; Pera, A.; Gevers, A.; et al. Development and validation of a new, simplified endoscopic activity score for Crohn's disease: The SES-CD. Gastrointest. Endosc. 2004, 60, 505-512. [CrossRef]

40. Best, W. Predicting the Crohn's disease activity index from the Harvey-bradshaw index. Inflamm. Bowel Dis. 2006, 12, 304-310. [CrossRef]

41. Williams, B.; Mancia, G.; Spiering, W.; Agabiti Rosei, E.; Azizi, M.; Burnier, M.; Clement, D.L.; Coca, A.; de Simone, G.; Dominiczak, A.; et al. 2018 ESC/ESH Guidelines for the management of arterial hypertension. Eur. Heart J. 2018, 39, 3021-3104. [CrossRef]

42. Van Bortel, L.M.; Laurent, S.; Boutouyrie, P.; Chowienczyk, P.; Cruickshank, J.K.; De Backer, T.; Filipovsky, J.; Huybrechts, S.; Mattace-Raso, F.U.; Protogerou, A.D.; et al. Expert consensus document on the measurement of aortic stiffness in daily practice using carotid-femoral pulse wave velocity. J. Hypertens. 2012, 30, 445-448. [CrossRef] [PubMed]

43. Mattace-Raso, F.; Hofman, A.; Verwoert, G.C.; Wittemana, J.C.; Wilkinson, I.; Cockcroft, J.; McEniery, C.; Yasmin Laurent, S.; Boutouyrie, P.; Bozec, E.; et al. Determinants of pulse wave velocity in healthy people and in the presence of cardiovascular risk factors: Establishing normal and reference values. Eur. Heart J. 2010, 31, 2338-2350.

44. Choi, Y.; Miura, M.; Nakata, Y.; Sugasawa, T.; Nissato, S.; Otsuki, T.; Sugawara, J.; Iemitsu, M.; Kawakami, Y.; Shimano, H.; et al. A common genetic variant of the chromogranin A-derived peptide catestatin is associated with atherogenesis and hypertension in a Japanese population. Endocr. J. 2015, 62, 797-804. [CrossRef] [PubMed]

45. Sciola, V.; Massironi, S.; Conte, D.; Caprioli, F.; Ferrero, S.; Ciafardini, C.; Peracchi, M.; Bardella, M.T.; Piodi, L. Plasma chromogranin a in patients with inflammatory bowel disease. Inflamm. Bowel Dis. 2009, 15, 867-871. [CrossRef] [PubMed]

46. Wagner, M.; Stridsberg, M.; Peterson, C.; Sangfelt, P.; Lampinen, M.; Carlson, M. Increased fecal levels of chromogranin A, chromogranin B, and secretoneurin in collagenous colitis. Inflammation 2013, 36, 855-861. [CrossRef]

47. Sugimoto, K. Role of STAT3 in inflammatory bowel disease. World J. Gastroenterol. 2008, 14, 5110. [CrossRef]

48. Chelakkot, C.; Ghim, J.; Ryu, S. Mechanisms regulating intestinal barrier integrity and its pathological implications. Exp. Mol. Med. 2018, 50, 103. [CrossRef]

49. Zhernakova, A.; Kurilshikov, A.; Bonder, M.; Tigchelaar, E.F.; Schirmer, M.; Vatanen, T.; Mujagic, Z.; Vila, A.V.; Falony, G.; Vieira-Silva, S.; et al. Population-based metagenomics analysis reveals markers for gut microbiome composition and diversity. Science 2016, 352, 565-569. [CrossRef] 
50. Egger, M.; Beer, A.; Theurl, M.; Schgoer, W.; Hotter, B.; Tatarczyk, T.; Vasiljevic, D.; Frauscher, S.; Marksteiner, J.; Patsch, J.R.; et al. Monocyte migration: A novel effect and signaling pathways of catestatin. Eur. J. Pharmacol. 2008, 598, 104-111. [CrossRef]

51. Figueiredo, V.; Yugar-Toledo, J.; Martins, L.; Martins, L.B.; de Faria, A.P.; de Haro Moraes, C.; Sierra, C.; Coca, A.; Moreno, H. Vascular stiffness and endothelial dysfunction: Correlations at different levels of blood pressure. Blood Press. 2011, 21, 31-38. [CrossRef]

52. Andersen, N.; Jess, T. Risk of cardiovascular disease in inflammatory bowel disease. World J. Gastrointest. Pathophysiol. 2014, 5, 359. [CrossRef]

53. Zanoli, L.; Cannavò, M.; Rastelli, S.; Di Pino, L.; Monte, I.; Di Gangi, M.; Boutouyrie, P.; Inserra, G.; Laurent, S.; Castellino, P. Arterial stiffness is increased in patients with inflammatory bowel disease. J. Hypertens. 2012, 30, 1775-1781. [CrossRef]

54. Nardone, M.; Incognito, A.V.; Millar, P.J. Evidence for pressure-independent sympathetic modulation of central pulse wave velocity. J. Am. Heart Assoc. 2018, 7, e007971. [CrossRef]

55. Dienno, F.A.; Jones, P.P.; Seals, D.R.; Tanaka, H. Age-associated arterial wall thickening is related to elevations in sympathetic activity in healthy humans. Am. J. Physiol. Heart Circ. Physiol. 2000, 278, H1205-H1210. [CrossRef]

56. Holwerda, S.W.; Luehrs, R.E.; DuBose, L.; Collins, M.T.; Wooldridge, N.A.; Stroud, A.K.; Fadel, P.J.; Abboud, F.M.; Pierce, G.L. Elevated muscle sympathetic nerve activity contributes to central artery stiffness in young and middle-age/older adults. Hypertension 2019, 73, 1025-1035. [CrossRef]

57. Maule, S.; Pierangeli, G.; Cevoli, S.; Grimaldi, D.; Gionchetti, P.; Barbara, G.; Riziello, F.; Stangellini, V.; Corinaldesi, R.; Campieri, M.; et al. Sympathetic hyperactivity in patients with ulcerative colitis. Clin. Auton. Res. 2007, 17, 217-220. [CrossRef]

58. Furlan, R.; Ardizzone, S.; Palazzolo, L.; Rimoldi, A.; Perego, F.; Barbic, F.; Bevilacqua, M.; Vago, L.; Bianchi Porro, G.; Malliani, A. Sympathetic overactivity in active ulcerative colitis: Effects of clonidine. Am. J. Physiol. Regul. Integr. Comp. Physiol. 2006, 290, R224-R232. [CrossRef] [PubMed]

59. Pei, Z.; Ma, D.; Ji, L.; Zhang, J.; Su, J.; Xue, W.; Chen, X.; Wang, W. Usefulness of catestatin to predict malignant arrhythmia in patients with acute myocardial infarction. Peptides 2014, 55, 131-135. [CrossRef] [PubMed]

60. Alam, M.J.; Gupta, R.; Mahapatra, N.R.; Goswami, S.K. Catestatin reverses the hypertrophic effects of norepinephrine in $\mathrm{H} 9 \mathrm{c} 2$ cardiac myoblasts by modulating the adrenergic signaling. Mol. Cell Biochem. 2020, 464, 205-219. [CrossRef] [PubMed]

61. Chen, Y.; Wang, X.; Yang, C.; Su, X.; Yang, W.; Dai, Y.; Han, H.; Jiang, J.; Lu, L.; Wang, H.; et al. Decreased circulating catestatin levels are associated with coronary artery disease: The emerging anti-inflammatory role. Atherosclerosis 2019, 281, 78-88. [CrossRef] [PubMed]

62. Lurz, E.; Aeschbacher, E.; Carman, N.; Schibli, S.; Sokollik, C.; Simonetti, G. Pulse wave velocity measurement as a marker of arterial stiffness in pediatric inflammatory bowel disease: A pilot study. Eur. J. Pediatr. 2017, 176, 983-987. [CrossRef] [PubMed]

63. Zanoli, L.; Boutouyrie, P.; Fatuzzo, P.; Granata, A.; Lentini, P.; Oztürk, K.; Cappello, M.; Theocharidou, E.; Tuttolomondo, A.; Pinto, A.; et al. Inflammation and aortic stiffness: An individual participant data meta-analysis in patients with inflammatory bowel disease. J. Am. Heart Assoc. 2017, 6, e007003. [CrossRef] [PubMed]

(C) 2020 by the authors. Licensee MDPI, Basel, Switzerland. This article is an open access article distributed under the terms and conditions of the Creative Commons Attribution (CC BY) license (http://creativecommons.org/licenses/by/4.0/). 